\title{
Computational fluid dynamics in the evaluation of hemodynamic performance of cavopulmonary connections after the Norwood procedure for hypoplastic left heart syndrome
}

\author{
Edward L. Bove, MD \\ Marc R. de Leval, MD \\ Francesco Migliavacca, $\mathrm{PhD}^{\mathrm{c}}$ \\ Gualtiero Guadagni, $\mathrm{PhD}^{\mathrm{c}}$ \\ Gabriele Dubini, $\mathrm{PhD}^{\mathrm{C}}$
}

Objective: Computational fluid dynamics have been used to study the hemodynamic performance of surgical operations, resulting in improved design. Efficient designs with minimal energy losses are especially important for cavopulmonary connections. The purpose of this study was to compare hydraulic performance between the hemi-Fontan and bidirectional Glenn procedures, as well as the various types of completion Fontan operations.

Methods: Three-dimensional models were constructed of typical hemi-Fontan and bidirectional Glenn operations according to anatomic data derived from magnetic resonance scans, angiocardiograms, and echocardiograms. Boundary conditions were imposed, and fluid dynamics were calculated from a mathematic code. Power losses, flow distribution to each lung, and pressures were measured at three predetermined levels of pulmonary arteriolar resistance. Models of the lateral tunnel, total cavopulmonary connection, and extracardiac conduit completion Fontan operations were constructed, and power losses, total flow distribution, vena caval and pulmonary arterial pressures, and flow distribution of inferior vena caval return were calculated.

Results: The hemi-Fontan and bidirectional Glenn procedures performed nearly identically, with similar power losses and nearly equal flow distributions to each lung at all levels of pulmonary arteriolar resistance. However, the lateral tunnel Fontan procedure as performed after the hemi-Fontan operation had lower power losses $(6.9 \mathrm{~mW}$, pulmonary arteriolar resistance 3 units) than the total cavopulmonary connection $(40.5 \mathrm{~mW})$ or the extracardiac conduit $(42.9 \mathrm{~mW})$, although the inclusion of an enlargement patch toward the right in the total cavopulmonary connection was effective in reducing the difference $(10.0 \mathrm{~mW})$. Inferior vena caval flow to the right lung was $52 \%$ for the lateral tunnel, compared with 19\%, 30\%, $19 \%$, and $15 \%$ for the total cavopulmonary connection, total cavopulmonary connection with right-sided enlargement patch, extracardiac conduit, and extracardiac conduit with a bevel to the left lung, respectively.

Conclusions: According to these methods, the hemi-Fontan and bidirectional Glenn procedures performed equally well, but important differences in energy losses and flow distribution were found after the completion Fontan procedures. The superior hydraulic performance of the lateral tunnel Fontan operation after the hemi-Fontan procedure relative to any other method may be due to closer to optimal caval offset achieved in the surgical reconstruction. 


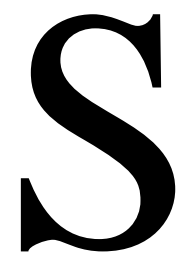

taged surgical reconstruction for hypoplastic left heart syndrome has become increasingly successful. ${ }^{1}$ As more survivors of the Norwood procedure reach their completion Fontan operations, increased scrutiny has been given to the methods used to construct the cavopulmonary connections. Because the energy available for pulmonary blood flow after a Fontan procedure is limited, streamlined cavopulmonary anastomotic designs are important to produce optimal clinical results. It is well established that minimizing energy losses through these connections is essential to achieve an efficient circulation, and a number of studies have used in vitro and computer flow modeling to evaluate the hydrodynamics of these operations in an effort to construct better designs. ${ }^{2-21}$ As de Leval and associates ${ }^{22}$ have demonstrated, power losses are increased in cavopulmonary connections where sharp angles are constructed, resulting in less optimal clinical outcomes. Sievers and colleagues ${ }^{9}$ emphasized that turbulence, particularly around the surgically constructed anastomoses, results in energy dissipation, flow disturbances, and maldistribution of pulmonary blood flow. Furthermore, these effects are likely to be magnified at increasing flow rates, which may contribute to the decreased exercise tolerance seen in many patients after the Fontan procedure.

The objectives of this study were twofold: (1) to compare the hydraulic performances of the hemi-Fontan procedure (HFP) and the bidirectional Glenn anastomosis (BDG) after the Norwood procedure for hypoplastic left heart syndrome and (2) to simulate the final Fontan procedure with the lateral tunnel (LT), total cavopulmonary connection (TCPC), and extracardiac conduit (ECC) techniques to evaluate the hemodynamic efficiencies and flow repartitions of these alternate procedures.

\section{Materials and Methods Mathematic Modeling}

Fluid dynamics in large vessels can be described by equations of mass and momentum conservation (Navier-Stokes equations) when the non-Newtonian features of blood can be ignored. ${ }^{23}$ These are partial derivative equations that can be analytically solved for simple geometries provided that boundary conditions are properly set. Computer flow dynamic (CFD) techniques allow the fluid dynamic field to be solved in most of the cases in which the analytic solution cannot be achieved. The first step in creating a 3-dimensional CFD model is to reproduce the geometry of the investigated region and divide the continuum into a number of simple volumes where the unknowns of the problem (pressure and velocity) will be evaluated. Geometric data are taken from cineangiograms, magnetic resonance (MR) images, and Doppler echocardiographic measurements. Imposition of the boundary conditions (velocity and pressure at the inlets and outlets of the model) is the second step. The mathematic code adopted will then calculate the fluid dynamic field.

\section{HFP Model}

A realistic 3-dimensional model of a typical HFP (Figure 1) was constructed on the basis of anatomic data from MR scans and cineangiocardiograms carried out on 3 patients after the Norwood procedure for hypoplastic left heart syndrome. The HFP modeled in this study was a true side-to-side amalgamation of the superior vena cava (SVC) and the pulmonary arteries, as opposed to separate end-to-side anastomoses of the divided SVC to the right pulmonary artery (RPA). Details of the adopted reconstruction method have been published previously elsewhere. ${ }^{17}$ A hybrid mesh was used for the fluid dynamics analysis, which included approximately 60,000 tetrahedral cells and 13,000 hexahedral cells. Mesh sensitivity was checked carrying out a steady-state simulation with a 130,000-cell model of the HFP, obtained through an adaptation method that was based on the evaluation of pressure gradient. The difference in the average pressure drop between the SVC inlet and the left pulmonary arterial (LPA) and RPA outlets from the two simulations was found to be less than $2 \%$. Blood flows in each pulmonary artery $\left(Q_{R P A}\right.$ and $\left.Q_{L P A}\right)$ were determined by two pressure drops in series, the first caused by the local geometry of the HFP and the second occurring through the corresponding lung resistances. To reproduce both effects in a coupled way, the pulmonary afterload was simulated by adding two small porous portions at the pulmonary arterial outlets. Downstream from them, the left atrial pressure $\left(P_{L A}\right)$ was imposed. Porous jump elements imposed an additional pressure drop $(\Delta P)$ :

$$
\Delta \mathrm{P}=\mu / \alpha \mathrm{Lv}
$$

where $v$ is the normal velocity, $\mu$ is the fluid viscosity, $\alpha$ is the medium permeability, and $L$ is the thickness of the porous portion. Left atrial pressure was assumed to be constant and equal to the mean pressure measured during cardiac catheterization $(4 \mathrm{~mm} \mathrm{Hg}$ ). Thus the left atrium was assumed to behave like a constantpressure reservoir. Data from cardiac catheterizations also supplied a range of pulmonary arteriolar resistances (PARs) for the model (1 to $4 \mathrm{~mm} \mathrm{Hg} /[\mathrm{L} \cdot \min )]$.

Further general assumptions for the models included rigid and impermeable vessel walls, homogeneous and Newtonian fluid ( $\rho=$ $1060 \mathrm{~kg} / \mathrm{m}^{3}, \mu=5 \times 10^{-3} \mathrm{~Pa} \cdot \mathrm{s}$ ), no-slip condition at the walls (velocity $=0$ ), and flat velocity profiles at the caval inlet section. The CFD solver package, Fluent 5.4 (Fluent Inc, Lebanon, NH), a commercial CFD package that is based on the finite volume method, was used to solve the transient Navier-Stokes equations.

SVC flow rate and time function were derived from an MR scan performed on a patient with a TCPC. ${ }^{16}$ Mean SVC flow rate was $1.08 \mathrm{~L} / \mathrm{min}$, the period of the cardiac cycle was 0.85 seconds, and the adopted PAR values were 1,3 , and $4 \mathrm{~mm} \mathrm{Hg} /(\mathrm{L} \cdot \mathrm{min})$. Equal lung resistances were assumed.

To evaluate the effects of the pulmonary afterload on cavopulmonary hemodynamics, a quantitative energetic index was also defined according to the control volume approach. The adopted control volume did not include the left and right lung resistances. Average fluid-energy dissipation (power loss, $\dot{W}$ ) was calculated at each time step and averaged during the cardiac cycle as follows:

$$
\dot{\mathrm{W}}=\sum \mathrm{Q}_{1}\left(\mathrm{P}_{\mathrm{i}}+\frac{1}{2} \rho \nu_{i}^{2}\right)-\sum \mathrm{Q}_{\mathrm{o}}\left(\mathrm{P}_{\mathrm{o}}+\frac{1}{2} \rho \nu_{0}^{2}\right)
$$

where $\rho, Q, P$, and $v$ (subscript $i$ indicating inlet SVC and subscript 

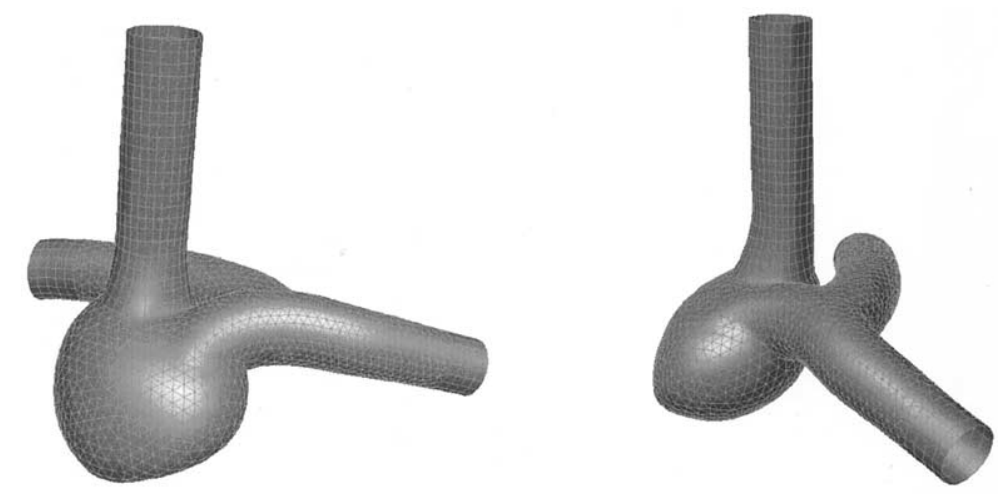

Figure 1. Three-dimensional model of typical HFP, based on anatomic data from MR scans and angiocardiograms.
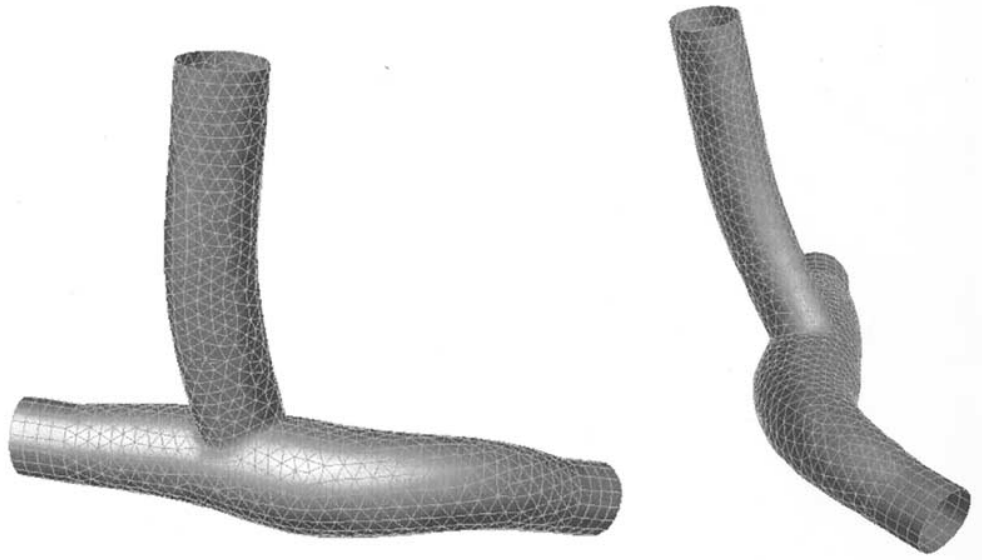

Figure 2. Three-dimensional model of typical BDG, based on anatomic data from MR scans and angiocardiograms.

$o$ indicating outlet LPA and RPA) are, respectively, blood density, volume flow rate, pressure, and mean velocities at inlet and outlets.

Flow distribution (FD) to the left lung was also calculated as a percentage of the inlet caval flow:

$$
\mathrm{FD}=\mathrm{Q}_{\mathrm{LPA}} / \Sigma \mathrm{Q}_{\mathrm{i}} \times 100 \%
$$

being the total pulmonary flow equal to the inlet caval flow $\left(\mathrm{Q}_{\mathrm{SVC}}\right.$ $\left.=\mathrm{Q}_{\mathrm{RPA}}+\mathrm{Q}_{\mathrm{LPA}}\right)$.

\section{BDG Model}

A geometric model of a typical BDG was developed, featuring the same pulmonary artery as for the HFP model as well as identical SVC length and inlet area. The model was constructed to simulate the surgical procedure by connecting the SVC directly to the pulmonary artery and removing the main pulmonary artery (Figure 2). The SVC inlet section is in the same geometric position as in the HFP model. Boundary conditions and fluid-energy dissipation were calculated as in the HFP model.

\section{Completion LT Fontan Model}

The completion Fontan LT model (Figure 3) was derived from the previous HFP model, removing the atrial patch and connecting the inferior vena cava (IVC) to the atriopulmonary anastomosis. Pul- satile SVC (mean $\left.\mathrm{Q}_{\mathrm{SVC}}=1.08 \mathrm{~L} / \mathrm{min}\right)$ and IVC (mean $\mathrm{Q}_{\mathrm{IVC}}=$ $1.21 \mathrm{~L} / \mathrm{min}$ ) inlet flows were adopted with a cardiac cycle of 0.85 seconds for the boundary conditions. The adopted time functions were those measured from MR scans as used in a previous study. ${ }^{16}$

Fluid-energy dissipation and assumptions were the same as in the HFP model, with the inclusion of the IVC quantities ( $i$ for inlet SVC and IVC). In this model the IVC flow contributions to each lung were quantified separately. By imposing different oxygen concentrations at the caval inlets and solving the oxygen mass conservation equation, outlet oxygen concentrations were obtained, which are related to the mass flows. Details of this method have been reported previously elsewhere. ${ }^{18}$

\section{TCPC and ECC Models}

The TCPC model was created by extending the IVC while keeping its inlet location the same as in the LT Fontan model. Several models were developed: a straight intra-atrial tunnel TCPC, an intra-atrial tunnel TCPC with an enlargement patch toward the RPA, a straight ECC to the pulmonary arteries, and an ECC with a bevel to the LPA. These were chosen to mimic the most commonly used clinical techniques. The models for the intra-atrial tunnels were chosen to mimic the technique of end-to-side anastomoses between the divided SVC and the RPA. The models are 
depicted in Figure 4. Boundary conditions, fluid-energy dissipation, and assumptions are as used in the LT Fontan model.

\section{Results \\ HFP and BDG Models}

Table 1 summarizes the averaged values in the cardiac cycle for the power losses, FD to the lungs, and inlet and outlet pressures. Figure 5 shows the path lines corresponding to an SVC injection for the HFP and BDG models. Table 1 demonstrates that power losses increase with progressive increases in PAR, with a consistently greater power loss in the BDG than in the HFP. Although to a lesser degree, this trend was also noted with regard to the vena caval pressures between the two procedures. However, power loss, FD, as well as SVC, LPA, and RPA pressures, were not substantially different between the two techniques, indicating similar hemodynamic performance. The effects of PAR on the hemodynamics in the HFP were addressed in a previous study. ${ }^{17}$

\section{Fontan Models}

Table 2 summarizes the averaged values in the cardiac cycle for the power losses, total (SVC plus IVC) FDs to the lungs, inlet and outlet pressures, and IVC FDs to the lungs separately for the various Fontan models. The lowest power losses were associated with the LT procedure at all levels of PAR, although adding a patch to the RPA in the TCPC lessened the differences. Power losses were higher with the straight TCPC, straight ECC, and beveled ECC models. For all Fontan models, total FDs were nearly equal in the two lungs. When IVC flow was quantified separately, however, only the LT Fontan maintained a balanced FD, with all other techniques favoring flow to the left lung. Both SVC and IVC pressures remained the lowest with the LT Fontan procedure, as did the differences between the inlet and outlet pressures. These differences were especially marked at higher levels of PAR. Figure 6 shows particle trajectory pictures at 0.3 seconds from the beginning of the cardiac cycle (period 0.85 second), corresponding to SVC and IVC injections for all Fontan models.

\section{Discussion}

Recent literature evaluating the hemodynamics of pediatric cardiac surgical procedures can be divided in two classes: experimental (in vitro) and numeric mathematic (CFD) modeling. One of the earliest in vitro experiments was performed by de Leval and associates, ${ }^{22}$ who reported visualization of flow through cavities and around corners in addition to measurements of energy losses across nonpulsatile cavities, corners, and stenoses. Those studies indicated the importance of streamlining and suggested an improvement in the hydrodynamic design of the Fontan circulation, the TCPC. In the following years researchers studied the hydrodynamics of this new connection and
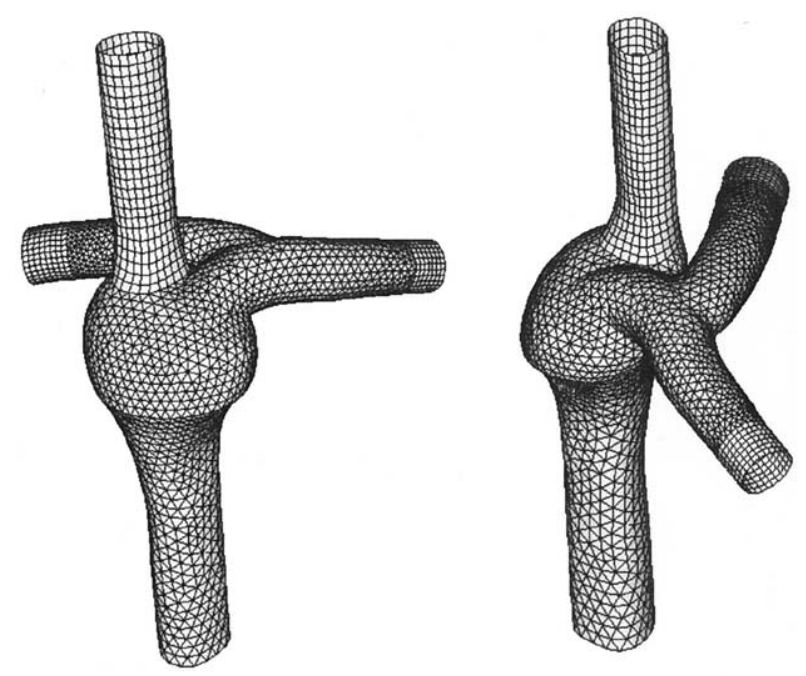

Figure 3. Model of completion LT Fontan, as performed after HFP by removal of intra-atrial patch.

compared it with the atriopulmonary connection. Low and

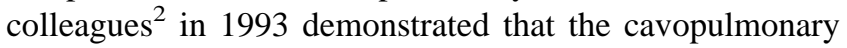
connection has much lower flow losses than does the atriopulmonary one. A study by Kim and colleagues ${ }^{3}$ in 1995 reached the same conclusions. Sharma and associates ${ }^{4}$ in 1996 and Ensley and colleagues ${ }^{5}$ in 1999 sought to evaluate the effects of offsetting and flaring cavopulmonary connections at varying pulmonary flow ratios to determine the optimal geometry of the connection. More recently, these investigators studied in vivo TCPC flow characteristics obtained by MR phase-contrast imaging and compared the results with those of the previous in vitro TCPC flow experiments. ${ }^{6}$ Similar studies were also performed by Gerdes and colleagues. ${ }^{7}$ Lardo and associates ${ }^{8}$ studied hydrodynamic efficiency between intra-atrial LT, extracardiac tunnel, and ECC, with or without caval vein offset, performed on explanted sheep heart preparations with an in vitro flow loop. With regard to the bidirectional cavopulmonary anastomosis, as far as we know only two works have appeared. Sievers and associates ${ }^{9}$ proposed a modification of the standard Norwood variant of cavopulmonary connection with an extended anastomosis on the basis of hydrodynamic results of two different in vitro models. Lardo and colleagues ${ }^{10}$ showed with an in vitro model that a bidirectional cavopulmonary anastomosis reduces fluidenergy dissipation in atriopulmonary connections, provides a physiologic distribution of total flow, and maintains some hepatic venous flow to each lung.

During the last 10 years, computational simulations of various surgical procedures have come into use in the clinical community. In particular, concerning the cavopulmonary connection, computer-generated models that are based 

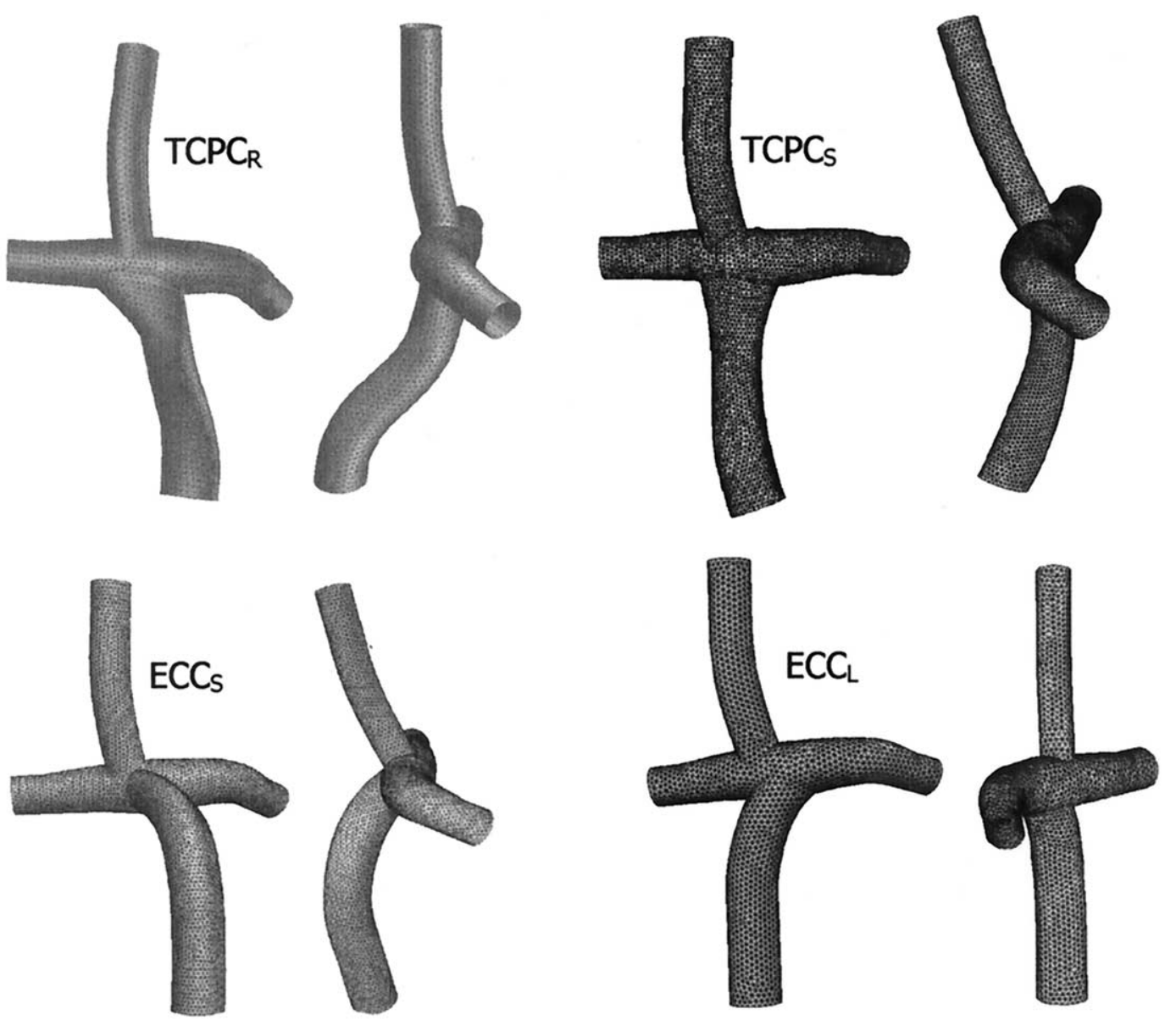

Figure 4. Various completion Fontan procedures, as performed after the BDG, illustrated in frontal and lateral views: TCPC with patch to right $\left(\right.$ TCPC $_{R^{\prime}}$ upper left), straight TCPC without patch $\left(T C P C_{S^{\prime}}\right.$ upper right), straight ECC without bevel $\left(E C C_{S^{\prime}}\right.$ lower left), and ECC with bevel to left $\left(E C C_{L^{\prime}}\right.$ lower right).

TABLE 1. Averaged values in the cardiac cycle for power losses, FD to the lungs, and pressures in the inlets and outlets of the models

\begin{tabular}{|c|c|c|c|c|c|c|}
\hline & \multicolumn{3}{|c|}{ HFP } & \multicolumn{3}{|c|}{ BDG } \\
\hline & 1 & 3 & 4 & 1 & 3 & 4 \\
\hline Power loss (mW) & 0.9 & 1.7 & 2.1 & 1.2 & 2.6 & 3.4 \\
\hline SVC flow LPA (\%) & 49.8 & 49.7 & 49.7 & 50.8 & 51 & 50.9 \\
\hline $\begin{array}{l}\text { SVC pressure } \\
(\mathrm{mm} \mathrm{Hg})\end{array}$ & 5.4 & 7.8 & 9 & 5.5 & 8.4 & 9.9 \\
\hline $\begin{array}{l}\text { LPA pressure } \\
(\mathrm{mm} \mathrm{Hg})\end{array}$ & 5.1 & 7.2 & 8.2 & 5.1 & 7.3 & 8.5 \\
\hline $\begin{array}{l}\text { RPA pressure } \\
(\mathrm{mm} \mathrm{Hg})\end{array}$ & 5.1 & 7.2 & 8.2 & 5.1 & 7.4 & 8.6 \\
\hline
\end{tabular}

Values are given for PARs (in millimeters of mercury per liter per minute) of 1,3 , and 4 for each model.

on the finite element method have demonstrated lower energy losses with a cavopulmonary rather than an atriopulmonary connection. ${ }^{11}$ Furthermore, asymmetry between the
SVC and IVC connections was demonstrated to improve the energetics. ${ }^{12,13,16,18,21}$ Local effects of pulsatile forward flow from the native pulmonary artery on blood flow repartition to the lungs have been investigated as well. ${ }^{14,15}$

The good performance of the LT Fontan procedure may have two fluid dynamics explanations: (1) the presence of a divergent shape in both the SVC and IVC anastomoses and (2) the anteroposterior offset of the two caval anastomoses with respect to the pulmonary arteries. The first explanation is supported by the results obtained in the ECC and TCPC models. Indeed, the inclusion of a lateral patch at the anastomosis of the IVC pathway to the pulmonary artery, which acts as a diffuser from the fluid dynamics point of view, allows pressure to recover and thus leads to better energetics performance. Following this line of thinking, the inclusion of an enlarged section in the SVC anastomosis of the BDG could improve the TCPC performance as well. This design solution was not taken into consideration in our study; changes to such a connection could affect the results sig- 
nificantly. In the LT Fontan model, the SVC shows a natural divergent section when entering the right atrium. This feature, absent in the Fontan procedures after a BDG, could explain the good performance of the LT Fontan design.

With respect to the second explanation, the ECC and TCPC models show an alignment of the venae cavae in the coronal plane, preventing any offset between the venae cavae and the pulmonary arteries. The only surgical option is offsetting the IVC and SVC along the pulmonary artery, which has been demonstrated as the best fluid dynamics solution, even if a competition or impingement with extensive recirculation areas and vortices is always present between the caval flows. ${ }^{4,5,12,13,18,21}$ In the LT Fontan model the competition between IVC and SVC flows is reduced because complete mixing occurs in the right atrium, with reduction of the velocities as a result of the divergent shapes of the caval ends. Furthermore, the better streamlining in the pulmonary arteries (Figure 5), without the presence of swirling and secondary flows, improves the performance of the LT Fontan.

\section{Clinical Inferences}

The findings of this study, the only CFD study, together with those of the recent simplified study by DeGroff and Shandas, ${ }^{20}$ on the Fontan circulation with pulsatile boundary conditions at the caval inlets, indicate that the important hemodynamic differences between the two commonly used methods of intermediate cavopulmonary connections, the HFP and the BDG, are only realized after the completion Fontan operation. There is no important difference between the performances of these techniques themselves, but a significant advantage was found for the completion LT procedure when performed after the HFP. Both types of completion Fontan techniques after the BDG, the TCPC and the ECC, are associated with higher energy losses, greater vena caval pressures, and less balanced IVC FD between the two lungs. The IVC flow into the pulmonary arteries (relevant for the hepatic factor issue) is evenly distributed in the
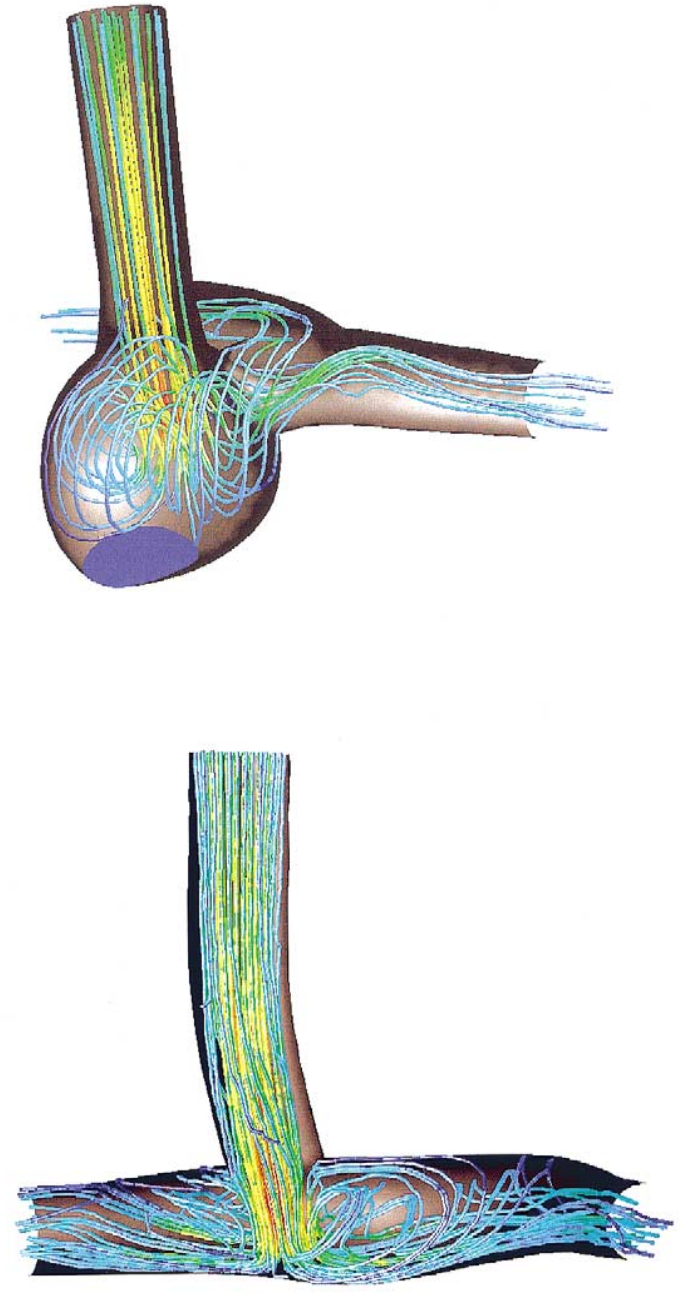

Figure 5. Path lines corresponding to SVC injection for HFP (upper panel) and BDG (lower panel) models. Lines are color coded with reference to velocity magnitude (red for high velocity, blue for low velocity).

TABLE 2. Averaged values in the cardiac cycle for power losses, FD to the lungs, pressures at the inlets and outlets of the models, and IVC blood distribution to the right lung

\begin{tabular}{|c|c|c|c|c|c|c|c|c|c|c|c|c|c|c|c|}
\hline & \multirow{2}{*}{\multicolumn{3}{|c|}{ LT }} & \multicolumn{6}{|c|}{ TCPC } & \multicolumn{6}{|c|}{ ECC } \\
\hline & & & & \multicolumn{3}{|c|}{ Straight } & \multicolumn{3}{|c|}{ Patch } & \multicolumn{3}{|c|}{ Straight } & \multicolumn{3}{|c|}{ Beveled } \\
\hline & 1 & 3 & 4 & 1 & 3 & 4 & 1 & 3 & 4 & 1 & 3 & 4 & 1 & 3 & 4 \\
\hline Power loss (mW) & 4.0 & 6.9 & 8.4 & 14.2 & 40.5 & 53.9 & 4.1 & 10.0 & 13.3 & 14.2 & 40.3 & 53.6 & 14.8 & 42.9 & 56.6 \\
\hline Total flow LPA (\%) & 50.4 & 49.9 & 50.1 & 50 & 49.6 & 50.0 & 49.1 & 49.9 & 49.8 & 49.5 & 49.8 & 49.9 & 52.0 & 52.1 & 52.1 \\
\hline IVC pressure $(\mathrm{mm} \mathrm{Hg})$ & 7.2 & 12.6 & 15.3 & 9.0 & 18.3 & 23.1 & 7.3 & 13.0 & 15.9 & 9.0 & 18.3 & 23.0 & 9.1 & 19.0 & 23.8 \\
\hline SVC pressure $(\mathrm{mm} \mathrm{Hg})$ & 7.2 & 12.7 & 15.3 & 9.0 & 18.4 & 23.1 & 7.3 & 13.0 & 15.9 & 9.0 & 18.3 & 23.1 & 9.2 & 19.0 & 23.8 \\
\hline LPA pressure $(\mathrm{mm} \mathrm{Hg})$ & 6.5 & 11.3 & 13.6 & 6.3 & 10.9 & 13.2 & 6.4 & 11.0 & 13.2 & 6.3 & 10.9 & 13.2 & 6.5 & 11.3 & 13.7 \\
\hline RPA pressure $(\mathrm{mm} \mathrm{Hg})$ & 6.5 & 11.3 & 13.6 & 6.4 & 11.0 & 13.3 & 6.5 & 11.2 & 13.5 & 6.4 & 11.0 & 13.7 & 6.3 & 10.8 & 13.0 \\
\hline IVC flow RPA (\%) & 49.3 & 51.6 & 52.2 & 19.1 & 18.7 & 21.3 & 30.7 & 30.2 & 29.8 & 19.2 & 19.2 & 18.7 & 15.6 & 15.5 & 15.5 \\
\hline
\end{tabular}

Values are given for PARs (in millimeters of mercury per liter per minute) of 1, 3, and 4 for each model. 

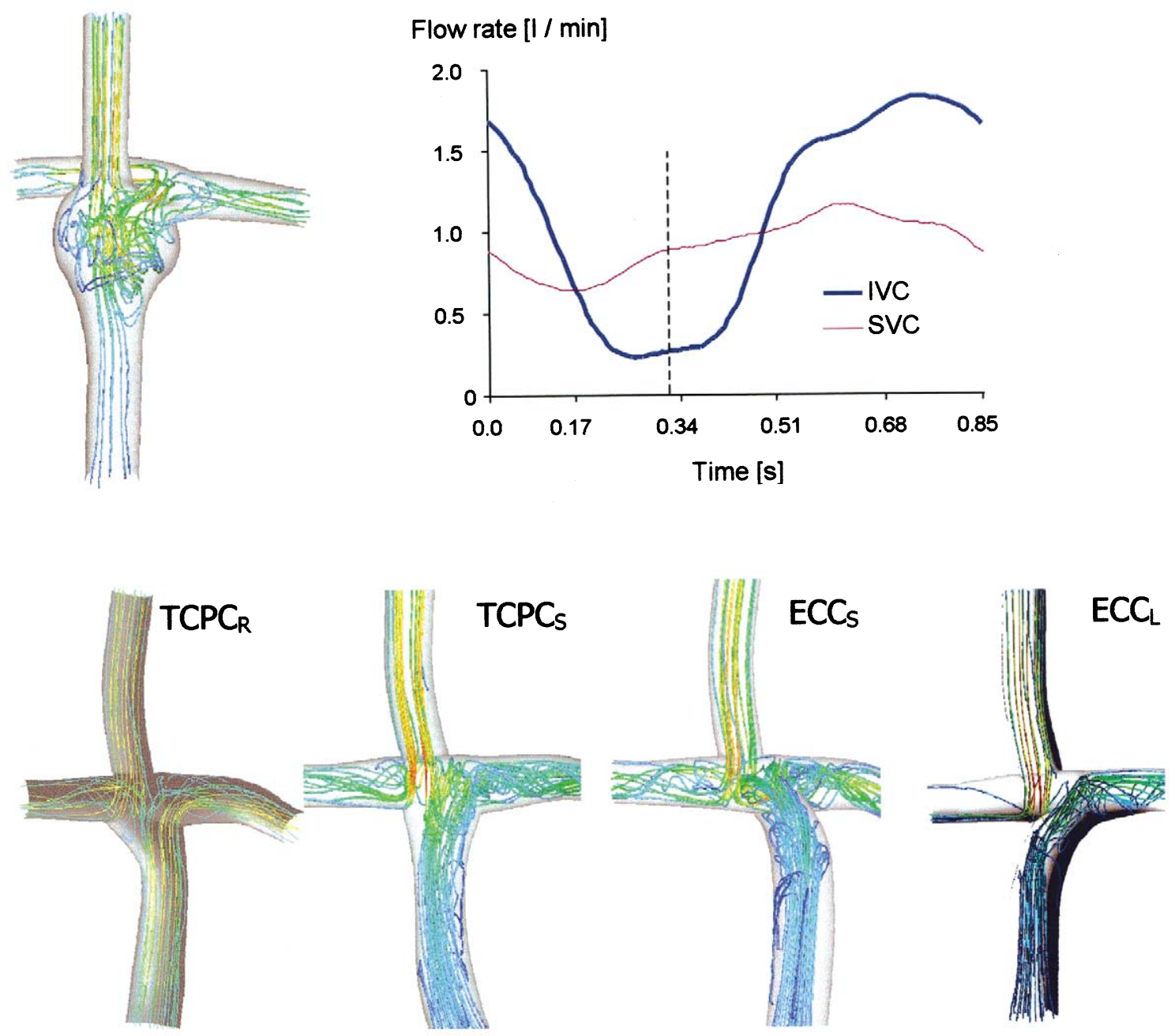

Figure 6. Path lines at 0.3 seconds (see time function plot, upper right pane/) corresponding to simultaneous SVC

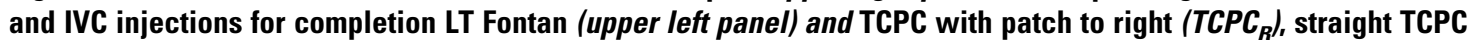
without patch $\left(T C P C_{s}\right)$, straight ECC without bevel (ECC $)_{s}$, and ECC with bevel to left (ECC $)$.

LT Fontan model, whereas the TCPC and ECC models exhibit preferential perfusion to the left lung, an effect most notable with the ECC model. Modeling the TCPC and the ECC with patches or beveling to either side reduced but did not eliminate these differences and could not guarantee a balanced IVC FD. Although the explanation for these findings is not immediately apparent from this study, the hemodynamic advantage realized from caval offset is probably involved. When either the TCPC or the ECC is done after a BDG anastomosis, caval offset is achieved by beveling the IVC portion of the connection to either the right or left lung. As demonstrated in this study, beveling the TCPC to the right conferred a significant advantage to the TCPC. Similarly, when the ECC was beveled toward the left lung, important differences were found in FD but not power losses. In contrast, the HFP provides a consistent caval offset in an anteroposterior direction because the IVC flow is directed anterior to that of the SVC. The LT connection is independent of that relationship. Although the exact performance characteristics of the TCPC and ECC procedures have important implications for efficiency of design, space constraints limit the surgeon's ability to offset the two caval return pathways as effectively as with the HFP. Obviously, this study did not test all possible variations of the TCPC or the ECC, and other, more favorable, outcomes may be possible with other models. In particular, the possible effects of enlargement of the right atrial-SVC junction, as well as the shape of the SVC-to-RPA anastomosis, were not addressed in this study.

The findings of this study are not necessarily intended to recommend a certain type of cavopulmonary connection solely on the basis of CFD techniques, because other patient-related and technical factors must also be taken into account. However, the importance of streamlined connec- 
tions is well established, and techniques associated with the lowest energy losses are likely to provide superior clinical outcomes during longer-term follow-up. Future studies evaluating the effects of respiration, increasing cardiac output, anomalies of venous connection, and other techniquerelated factors will be of importance in designing the optimal procedure.

\section{References}

1. Bove EL, Lloyd TR. Staged reconstruction for hypoplastic left heart syndrome: contemporary results. Ann Surg. 1996;224:387-95.

2. Low HT, Chew YT, Lee CN. Flow studies on atriopulmonary and cavopulmonary connections of the Fontan operations for congenital heart defects. J Biomed Eng. 1993;15:303-7.

3. Kim YH, Walker PG, Fontaine AA, Panchal S, Ensley AE, Oshinski $\mathrm{J}$, et al. Hemodynamics of the Fontan connection: an in-vitro study. J Biomech Eng. 1995; 117:423-8.

4. Sharma S, Goudy S, Walker P, Panchal S, Ensley A, Kanter K, et al. In vitro flow experiments for determination of optimal geometry of total cavopulmonary connection for surgical repair of children with functional single ventricle. J Am Coll Cardiol. 1996;27:1264-9.

5. Ensley AE, Lynch P, Chatzimavroudis GP, Lucas C, Sharma S, Yoganathan AP. Toward designing the optimal total cavopulmonary connection: an in vitro study. Ann Thorac Surg. 1999;68:1384-90.

6. Sharma S, Ensley AE, Hopkins K, Chatzimavroudis GP, Healy TM, Tam VK, et al. In vivo flow dynamics of the total cavopulmonary connection from three-dimensional multislice magnetic resonance imaging. Ann Thorac Surg. 2001;71:889-98.

7. Gerdes A, Kunze J, Pfister G, Sievers HH. Addition of a small curvature reduces power losses across total cavopulmonary connections. Ann Thorac Surg. 1999;67:1760-4.

8. Lardo AC, Webber SA, Friehs I, del Nido PJ, Cape EG. Fluid dynamic comparison of intra-atrial and extracardiac total cavopulmonary connections. J Thorac Cardiovasc Surg. 1999;117:697-704.

9. Sievers HH, Gerdes A, Kunze J, Pfister G. Superior hydrodynamics of a modified cavopulmonary connection for the Norwood operation. Ann Thorac Surg. 1998;65:1741-5.

10. Lardo AC, Webber SC, Iyengar A, del Nido PJ, Friehs I, Cape EG. Bidirectional superior cavopulmonary anastomosis improves mechanical efficiency in dilated atriopulmonary connections. J Thorac Cardiovasc Surg. 1999;188:681-91.

11. Van Haesdonck JM, Mertens L, Sizaire R, Montas G, Purnode B, Daenen W, et al. Comparison by computerized numeric modeling of energy losses in different Fontan connections. Circulation. 1995;92(9 Suppl):II322-6.

12. Dubini G, de Leval MR, Pietrabissa R, Montevecchi FM, Fumero R. A numerical fluid mechanical study of repaired congenital heart defects: application to the total cavopulmonary connection. J Biomech. 1996;29:111-21.

13. de Leval MR, Dubini G, Migliavacca F, Jalali H, Camporini G, Redington A, et al. Use of computational fluid dynamics in the design of surgical procedures: application to the study of competitive flows in cavo-pulmonary connections. J Thorac Cardiovasc Surg. 1996;111: 502-13.

14. Migliavacca F, de Leval MR, Dubini G, Pietrabissa R. A computational pulsatile model of the bidirectional cavopulmonary anastomosis: the influence of pulmonary forward flow. J Biomech Eng. 1996;118: $520-8$.

15. Migliavacca F, Dubini G, Pietrabissa R, de Leval MR. Computational transient simulations with varying degree and shape of pulmonic stenosis in models of the bidirectional cavopulmonary anastomosis. Med Eng Phys. 1997;19:394-403.

16. Migliavacca F, Kilner PJ, Pennati G, Dubini G, Pietrabissa R, Fumero $\mathrm{R}$, de Leval MR. Comparison between computational fluid dynamic and magnetic resonance analyses on a case of total cavopulmonary connection. IEEE Trans Biomed Eng. 1999;46:393-9.

17. Guadagni G, Migliavacca F, Dubini G, Bove EL. Effects of pulmonary afterload on the hemodynamics after the hemi-Fontan procedure. Med Eng Phys. 2001;23:293-8.

18. Migliavacca F, de Leval MR, Dubini G, Pietrabissa R, Fumero R. Computational fluid dynamic simulations of cavopulmonary connections with an extracardiac lateral conduit. Med Eng Phys. 1999;23: 187-93.

19. Ryu K, Healy TM, Ensley AE, Sharma S, Lucas C, Yoganathan AP. Importance of accurate geometry in the study of the total cavopulmonary connection: computational simulations and in vitro experiments. Ann Biomed Eng. 2001;29:844-53.

20. DeGroff C, Shandas R. Designing the optimal total cavopulmonary connection: pulsatile versus steady flow experiments. Med Sci Monit. 2002;8:MT41-5.

21. Khunatorn Y, Mahalingam S, DeGroff CG, Shandas R. Influence of connection geometry and SVC-IVC flow rate ratio on flow structures within the total cavopulmonary connection: a numerical study. $J$ Biomech Eng. 2002;124:364-77.

22. de Leval MR, Kilner P, Gewillig M, Bull C. Total cavopulmonary connection: a logical alternative to atriopulmonary connection for complex Fontan operations. Experimental studies and early clinical experience. J Thorac Cardiovasc Surg. 1988;96:682-95.

23. Fung YC, Biomechanics: Circulation. New York: Springer-Verlag; 1997. 